\title{
ADVANCES IN SOLAR ENERGY RESOURCE ASSESSMENT FOR CHILE
}

\author{
Rodrigo A. Escobar ${ }^{1}$, Samuel Luna de Abreu2 ${ }^{2}$, Alberto A. Ortega ${ }^{1}$, and Fernando Ramos Martins ${ }^{3}$ \\ ${ }^{1}$ Pontificia Universidad Católica de Chile, Santiago, Chile \\ 2 Instituto Federal de Santa Catarina, Florianópolis, Brazil \\ ${ }^{3}$ Instituto Nacional de Pesquisas Espaciais INPE, Sao Jose dos Campos, Brazil
}

\section{The Need for Solar Energy Data in Chile}

Renewable Energy promotion in Chile has obtained institutional support by means of a law that mandates a renewable energy quota of up to $10 \%$ of the electrical energy generated, which must be met by 2024 , with public announcements already being made that would modify this goal in order to achieve $20 \%$ of power generation by 2020 from renewable energy. This plan has sparked interest in introducing renewable energy systems to the country's electricity system. Solar energy is currently at the initial stages of market penetration, with several projects being announced including PV, CSP, and industrial heat supply plants. However, strong barriers still exists due to the absence of a valid solar energy database, adequate for energy system simulation and planning activities. Previous reports by the authors in both the Johannesburg 2009 SWC and Renewable Energy Journal (Ortega et al, 2009 and 2010) identified several databases of solar radiation which are available for Chile and discussed their merits and shortcomings. It has been found that significant deviation exists between sources, and that all ground station measurements display unknown uncertainty levels, thus highlighting the need for a proper, country-wide long-term resource assessment initiative. However, the solar energy levels throughout the country can be considered as high, and it is thought that they are adequate for energy planning activities -although not yet for proper power plant design and dimensioning. As a general conclusion, the previous work by the authors demonstrated that although for Chile there are several databases of ground measurements, a weather simulation model, and satellite-derived data, none of these data sources are completely valid and therefore a nationwide effort of resource assessment was needed.

As context, it is possible to mention that solar radiation data for large spatial regions can be obtained from ground station networks that provide discrete data points from which a continuous map can be obtained by means of a proper interpolation scheme. In addition, surface radiation can be estimated by satellite data processing. The latest Brazilian Solar Atlas (Pereira et al. 2006), for example, combines both measurement techniques in order to obtain data with low uncertainty levels. Pyranometer-based measurements from ground stations typically have lower uncertainty levels that satellite-derived data obtained by radiative transfer models, although this cannot be guaranteed for locations in between stations for data that has been computed by means of interpolation schemes. However, it has been shown that uncertainty levels for ground stations data are higher than satellite-derived measurements whenever the distance between stations is larger than $35 \mathrm{~km}$ (Perez et al 1997, Zelenka et al 1999 ), and thus, a sensible resource assessment campaign will try to use satellite-derived irradiance for ample terrain coverage, at the same time as the use of ground stations for monitoring and validation purposes. As reference regarding proper time periods for measurement campaigns, the temporal variability of solar irradiance indicates that 5-year data sets can help determine the long-term average solar radiation with a fair degree of accuracy (estimated to be slightly larger than 5\%), but do not contain enough information to accurately represent year-to-year variability. A 15 -year data set can show inter annual patterns and trends, although statistically these variations are complex and do not follow a simple bell shaped curve of a random distribution. However, as mentioned by Pitz-Paal (2007), a long term accurate average can be obtained by this data. The characteristics of solar irradiance can be described with a high degree of statistical confidence by analyzing 30-year data sets (Oregon University, 1999). The current efforts in assessing the solar resource in Chile aim to produce databases that satisfy the previously stated conditions. 
This report updates the previous article by presenting the advances made during the last two years regarding solar energy resource assessment in Chile. A new network of ground stations aiming to achieve BSRN standard of operation is being deployed in the country by the authors that provides developers, researchers and policymakers with good quality data. The location of this network is presented along the first measurement results for different climates. A second network composed of rotating shadowband radiometer devices (RSBR) is also being deployed by the authors in isolated locations of scientific interest such as high altitude places, salt lakes, snow covered terrain, and others. An existing network of ground stations was deployed by government agencies using non-standard methods that have introduced errors in the measurements, as was discussed in previous reports. The data produced by these stations has been reprocessed in order to improve DNI estimations. Finally, a new satellite estimation model is being developed by the authors. The Chile-SR model builds upon the Brazil-SR model partially developed by researchers at the Instituto Nacional de Pesquisas Espaciais (INPE) of Brazil, and introduces different treatments for the meteorological variables and the effective cloud cover computations. The report presents results and comparisons for all four data sources. Partial validation of the Chile-SR model is also presented, in which becomes apparent that the model is properly estimating solar radiation for temperate climates, although further refinement of the methodology is needed for desert areas with clear skies, where improved effective cloud cover estimates and better atmospheric aerosol modeling are needed. In what follows, we will first describe each database of ground station measurements that are available to the public. The satellitebased Chile-SR model is then described. Finally, a comparison between the data produced by different sources is attempted when possible.

\section{Available Data}

We consider that solar radiation data for Chile is available to the public if the following conditions are met: the database existence is known, no fee has to be paid in order to access the data, and the data can be accessed either through a website or by direct contact with the owners. As previously reported, there are several such databases in Chile, belonging to the National Meteorological Agency (Dirección Meteorológica de Chile - DMC), Technical University Santa María (through its Solar Evaluation Laboratory which includes the National Solarimetric Archive - NSA), the Chilean National Energy Commission (CNE Comisión Nacional de Energía) in collaboration with the German cooperation agency GiZ, and the Pontifical Catholic University of Chile (UC) in collaboration with FONDEF (Fondo de Desarrollo Científico y Tecnológico - Scientific and Technological Development Fund). DMC operates a network of 18 meteorological stations containing pyranometers with coverage from 1988 to date; the NSA considers ground station measurements from 89 stations along the Chilean territory, which includes data from as early as 1961; the GiZ-CNE network is composed of 7 stations that have been deployed since 2008, and the UCFONDEF network includes 5 stations designed under BSRN guidelines, and 7 RSBR units; all deployed or being deployed since mid-2010. In what follows we will briefly describe each network. Additional details of each but the UC-FONDEF networks and data sources can be found in Ortega et al. (2010).

\subsection{NSA data}

This database is under custody of the Chilean National Solarimetric Archive, located at Universidad Técnica Federico Santa María, Valparaiso. The stations were not operated continuously, but from as much as 21 years to a minimum of 3 months (Cáceres, 1984). The archives of measurements from the stations include both complete and incomplete years. Therefore, the data from the archive might have high yet unquantifiable uncertainties associated to the measurement period, plus the uncertainty which is inherent to the use of the actinographs (pyranographs) and Campbell-Stokes devices which were used in the measurements. In addition to the uncertainty inherent to the device and the time period of measurement, a human operator had to manually measure and integrate the produced data, as seen in Fig. 1. Unfortunately there aren't maintenance registries for any of the stations, and thus it is not possible to evaluate the calibration status and instrument condition. The data is available in two forms: processed data spanning 1961 to 1983 that has been published as tables in a book (Sarmiento, 1984), and unprocessed data available by direct request to the NSA (contact given in the references). The processed data includes monthly means for each location, with the 
addition of hourly data supplied for a typical day of each month obtained from a clear-day model. No information is given in the book regarding statistical procedures utilized to construct the monthly means, and no description is given about the model utilized to construct the hourly data. An interpolation method was utilized in order to create a contour map of monthly means for the whole Chilean territory.
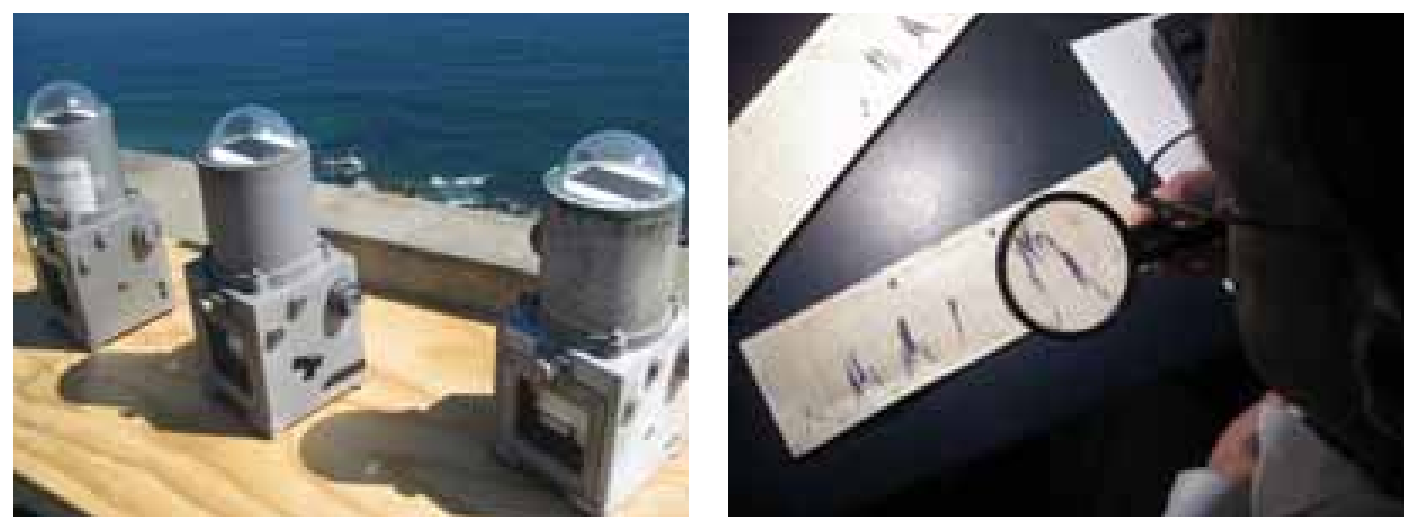

Figure 1: Actinographs of the NSA (left), and Mr. Roberto Sotta of the NSA processing results.

The unprocessed data includes hourly values for the period in which each station was active, and can be supplied in xls format. This data has been processed by the authors in collaboration with the NSA, determining long-term averages, trends, monthly means, and typical years, although the results are yet to be published.

\subsection{DMC data}

The Dirección Meteorológica de Chile has a series of pyranometers located at meteorological stations covering the main climate regions of the country. As of 2011 a total of 18 meteorological stations with pyranometers have been reported. A number of them are already decommissioned due to maintenance costs, and some others have been switched to different locations The data can be requested directly to the DMC at their website www.meteochile.cl, and is freely available to the public at a modest fee that covers processing costs. The data is integrated in 10 minute intervals by pyranometers covering the 0.285 to $2.8 \mu \mathrm{m}$ spectral range, and is presented as hourly-integrated irradiation $\left(\mathrm{Wh} / \mathrm{m}^{2}\right)$ from which hourly mean irradiation $\left(\mathrm{W} / \mathrm{m}^{2}\right)$ is easily computed, spanning complete months or years as the customer requests, and is provided in xls worksheets. The pyranometers are properly maintained and calibrated by DMC personnel. The DMC is also the custodian of an absolute cavity pyrheliometer traced to the world reference at Davos.

\subsection{CNE-GiZ ground stations}

The Chilean CNE requested the German cooperation agency GTZ to conduct a series of Renewable Energy assessments, including solar energy potential. A network of seven ground stations has been deployed since 2008, all located in the northern part of Chile in the Atacama Desert. The stations utilize three Kipp\&Zonnen CMP11 pyranometers, a datalogger with a scan rate of 1 or 2 seconds which saves data in 10minute averages, also measuring wind speed and temperature sensors.

Figure 2: View of a CNE-GiZ station.

The stations are operated and maintained by independent local

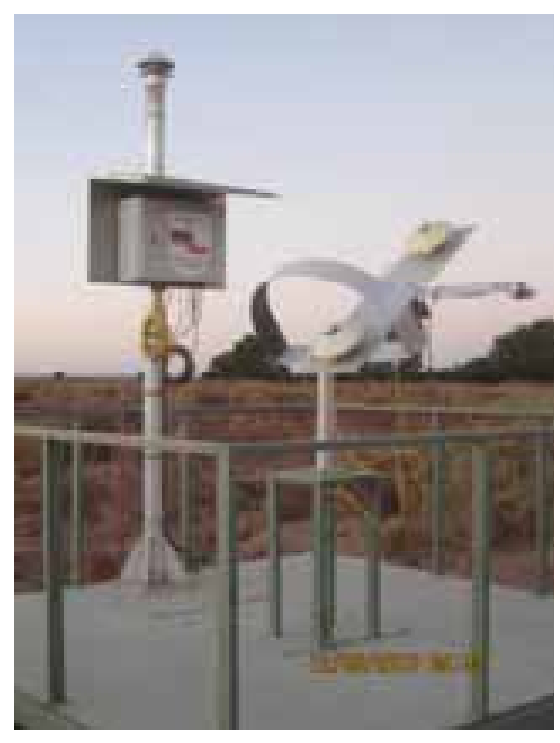
consulting companies hired by GiZ. Maintenance and data recovering from the datalogger are performed 
with monthly frequency. One pyranometer measures global horizontal irradiance, and the remaining two are mounted into a east-west one-axis solar tracker intended for use in PV systems. The first tracker-mounted pyranometer measures global irradiance in tracking mode, and the second measures diffuse irradiance in

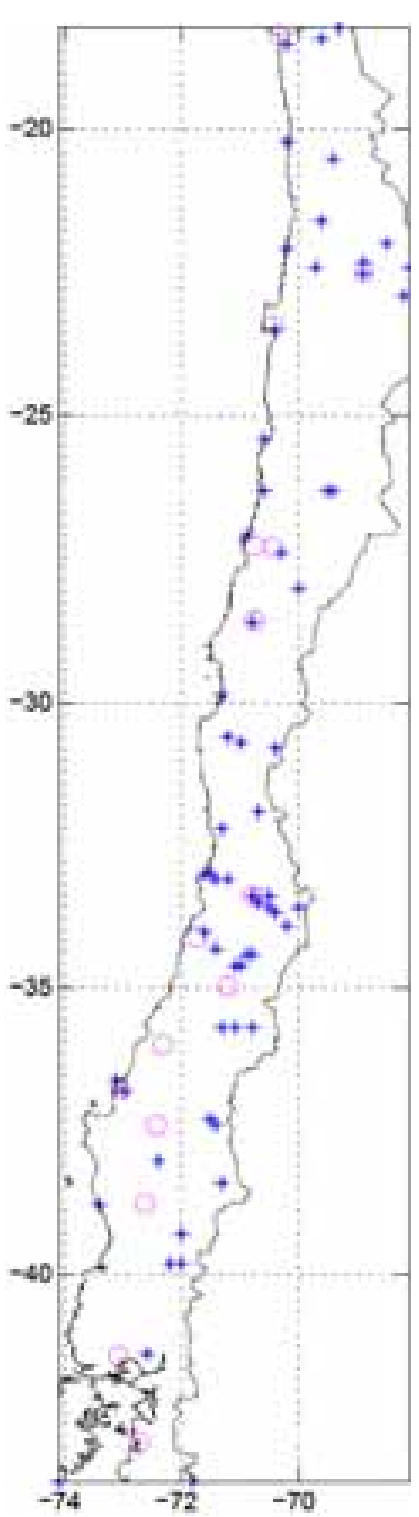
tracking mode as it is covered by a shadow ring. This way, an estimation of direct irradiance in the tracking plane is made by subtracting the diffuse from the global reading. Figure 2 shows a view of the station located at San Pedro de Atacama. The shortcomings of the design and operation of these stations have been discussed elsewhere (Ortega et al 2010), where it was argued that the validity of the measurements is severely restricted particularly in what is related to the direct irradiance values being computed. However, GiZ and CNE state that the objective of this measurement campaign is not to generate scientific quality data, but rather obtain consistent, trustable and comparable data in order to enhance knowledge of available radiation in northern Chile (www.cne.cl). Five of the GiZ-CNE stations have had their data reprocessed by Fraunhofer ISE by reviewing the data and computing the DNI values for 10-minute and hourly averages. It was determined that a $\pm 18.2 \%$ uncertainty exists in the computed DNI for hourly values of DNI, with a $\pm 8.7 \%$ uncertainty for global horizontal irradiance. Installation reports are available at the website www.cne.cl, as well as raw and re-processed data in pdf format of monthly reports and xls worksheets. Calibration status of these stations is unknown.

Figure 3: Location of ground stations in Chile.

\subsection{UC-FONDEF stations}

Starting in January 2010, a research project directed by the authors and financed through FONDEF grant D08i1097 is deploying a network of 12 ground stations, of which 5 are designed and operated under BSRN standards, and the remaining 7 are of three different configurations of RSBR. The stations designed following BSRN standards are composed of Kipp\&Zonnen Solys 2 trackers, sun sensors, CMP 11 and 21 pyranometers, heating and ventilation units, CGR6 pyrgeometers, CHP1 pyrheliometers, CUV4 UV meters, and also temperature, atmospheric pressure, relative humidity, wind speed and direction sensors, all connected to Campbell CR1000 dataloggers, with power supplied from the grid. Both scan and save rates follow BSRN guidelines, as well as the maintenance activities.

The RSBR devices can have any of three different configurations. The basic configuration includes an Irradiance Inc. RSBR2 device, composed by a Licor radiometer, the motor controller and rotating shadow band, temperature, atmospheric pressure, relative humidity, wind speed and direction sensors, all connected to Campbell CR1000 dataloggers, with power supply from a small-scale PV system. A second configuration lacks all meteorological sensors, and is used in locations that have a meteorological station in order to avoid repeated sensors. A third configuration is similar to the first one, with the addition of a CMP11 pyranometer for a redundant measurement of global horizontal radiation. This is used in sites where radiation conditions are particularly interesting and which have personnel readily available for maintenance and cleaning of the CMP11 device. Figure 4 shows two different configurations of the RSBR, one BSRN-designed station deployed in the field, and the Solar Evaluation Lab at UC in Santiago where all stations are received previous to field deployment. One BSRNdesigned and one RSBR are permanently measuring at this location. These stations have the objective of supplying data that satisfies international standards and criteria for design, operation and maintenance, thus 
providing high quality data for project developers and policymakers, and for the Chile-SR satellite estimation model described in a following section. Calibration certificates for these stations are still valid, and it is planned to trace them to the world reference as suggested by international standards with the proper frequency. Data qualification algorithms are being transferred from INPE to UC and will allow analyzing the quality of data being generates in the stations.
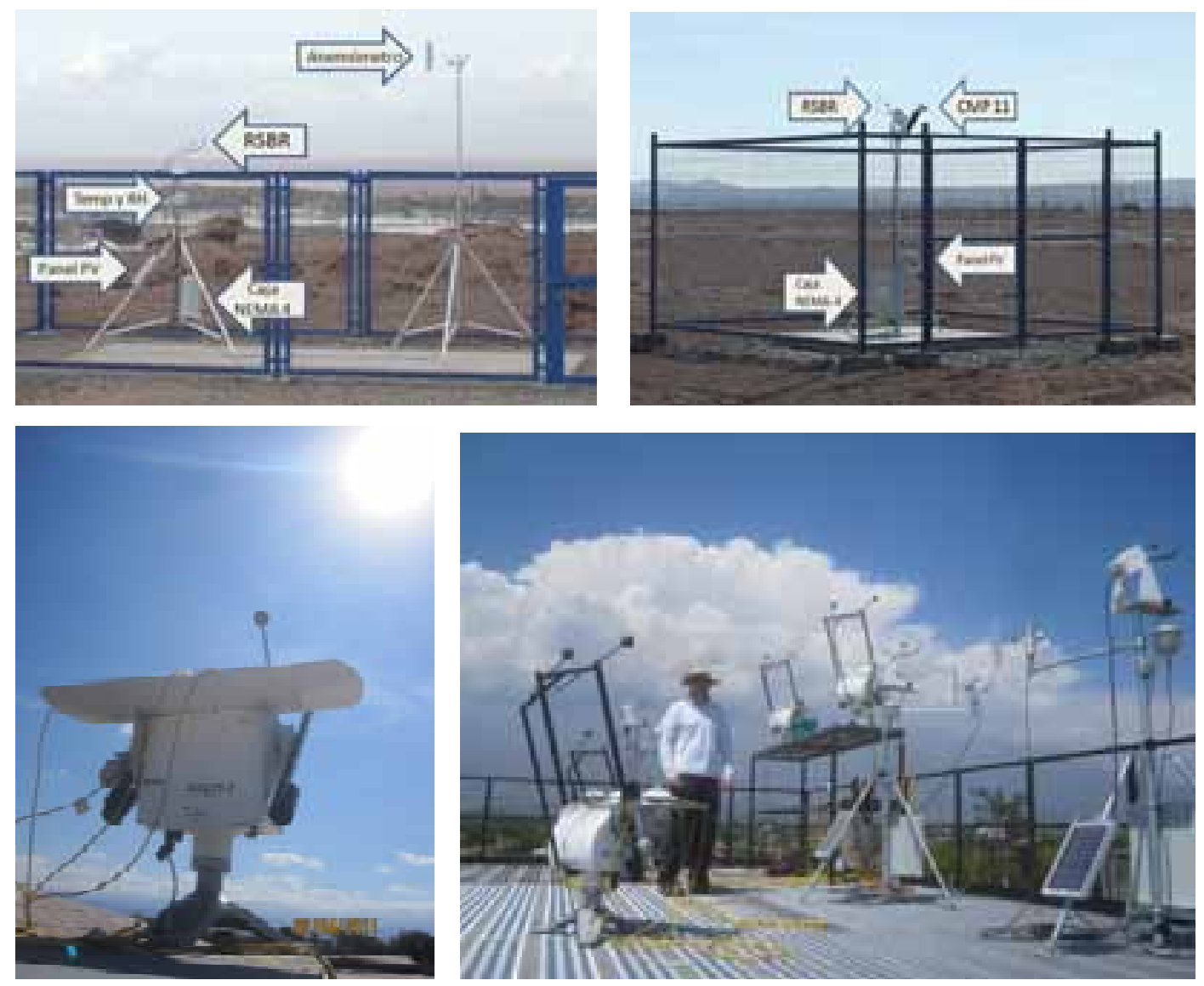

Figure 4: Two different configurations for RSBR stations (top); one BSRN-standard station (bottom left); author Alberto Ortega at the UC Solar Evaluation Lab in Santiago (bottom right) where both BSRN-standard and RSBR radiometers are tested prior to field deployment. Nice clouds over the Andes Mountains at the back.

\subsection{Comparing data from different sources}

Considering that there are at least four different sources of solar radiation data that are available to the public and also some others that are not freely available, then it stands to reason as logical to compare the data from each of these databases in order to analyze a site of particular interest. A site where solar projects are being developed is El Tesoro, located in the Antofagasta region in northern Chile. This place has exceptionally good conditions for the development of solar energy projects: apparently it receives large amounts of radiation; has more than 350 clear days a year; it is near a mining facility that has a large power and heat consumption, and is located in ample, flat terrain. In particular, this place is considered in Chile as one of the best, if not the best, for solar energy project development. Figure 5 displays a compilation of solar radiation data for El Tesoro: "MET" stands for data produced by a meteorological station located at the mining facility which includes a 10-year old Licor sensor that has measured for a similar period; "Meteo" corresponds to data obtained from the Meteonorm software; "RSBR" stands for data produced by one of the UC-FONDEF rotating shadowband devices deployed in late 2008; "ANS" refers to NSA data processed by the authors, and "SAT" stands for satellite-derived data from the GL1.2 model as described in Ortega et al (2010), which covers 1995 to 2005. The image on the left of Fig. 5 displays the location of El Tesoro in northern Chile 
superimposed to a "SAT" map of yearly mean global horizontal radiation. The data in Fig. 5 corresponds to monthly averages of daily radiation, in $\mathrm{kWh} / \mathrm{m}^{2}$. Neither the GiZ-CNE nor the DMC networks have a radiometer deployed in a location close to El Tesoro.
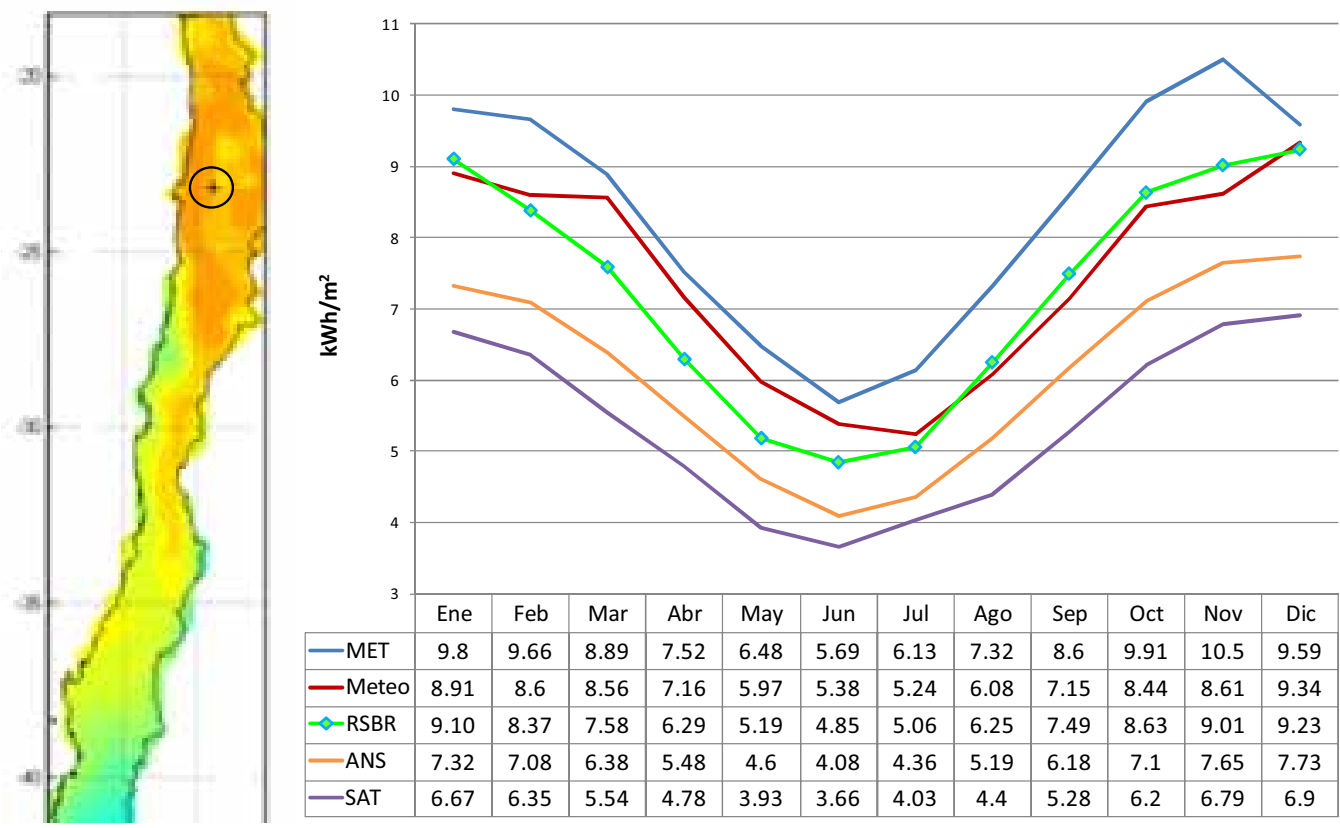

Figure 5: Location of El Tesoro in northern Chile (left), and compilation of available solar radiation data for the place (right).

Comparison of all data sources for this location indicate that yearly global horizontal radiation could be anywhere from 1960 to $3050 \mathrm{kWh} / \mathrm{m}^{2}$, that is, a $40 \%$ difference exists between the maximum and minimum radiation levels, and this without considering any uncertainty on the data related to measurement quality or long-term averages. In particular, it can be observed that recent measurements (RSBR) performed by a calibrated sensor in a properly maintained station have produced data that locates cleanly at the middle of the range enveloped by the extreme high (MET) and extreme low (SAT) radiation data. Both the ANS and SAT data severely underestimate radiation levels at El Tesoro, while MET and Meteo to a lesser degree overestimate the radiation level.

The questions that immediately arise by observing the data are: How can any project be developed with such uncertainty in the data?, and what can be done to improve this situation?. Observation of this hard reality led the authors to develop the nationwide resource assessment initiative funded by FONDEF known as Chile-SR satellite estimation model.

\section{Chile-SR Satellite-based Estimation Model}

It has been noted before that ground measurement campaigns, although accurate, are expensive and prone to equipment failure. In addition, poor maintenance leads to data of higher uncertainty, while the cost and length of a campaign prevents deploying the large number of ground stations needed to properly cover an extended geographical region such as a country. Satellite estimation of solar radiation at the earth surface combines several advantages over ground measurements that make it a preferred method for identifying sites with high potential before deciding to commit to a ground measurement campaign. Satellite estimation is cheaper yet sufficiently accurate, and it covers a large geographical area with adequate spatial and temporal resolution; however, its basic methodology remains a combination of remote sensing and numerical algorithm, and thus needs validation by ground station data in order to be considered as quality data useful for project development and policy making. Moreover, a methodology that relies on modeling the atmospheric transmittance has to adapt to the different climate conditions that can be present in a geographic 
region such as a country or continent, and therefore it is desirable to develop region-specific methodologies that are properly validated. Considering this, the authors aimed at developing a satellite estimation model that could produce accurate and low uncertainty data for Chile by taking into account the different climatic characteristics that the country displays, with enough spatial and temporal resolution to be used for project development. The Chile-SR model is being developed by the authors as a modification of the existing BrasilSR model developed by INPE within the SWERA project (swera.unep.net). The model takes the basic Brasil-SR algorithm and modifies it in order to create an adaptation especially suited for the largely different conditions that Chile presents with respect to Brazil. In particular, the northern region of Chile is the Atacama Desert, characterized by the absence of clouds throughout the year which result in high radiation levels reaching the surface. Moving further south, first a Mediterranean climate is found in the country's center region, which gives way to a cold forest region in southern Chile. Moreover, the country is a narrow strip of land located between the Pacific Ocean and the Andes Mountains. Therefore, it is possible to roughly divide the country into nine different regions, as defined by a matrix composed of north/center/south and coast/interior/mountains regions. The Chile-SR model had to accommodate for all these combinations, each of which has a different atmospheric profile, cloud cover, and topography.

According to this, the Chile-SR model is made specific for the conditions of Chile by including updated altitude-corrected weather data (temperature, relative humidity, and atmospheric pressure), topography, and albedo. GOES images for visible and IR channels are used as input to first classify cloud types, and then determine an effective cloud cover. The atmospheric radiative transfer algorithm that Brasil-SR utilizes is left untouched but for the input data and the effective cloud cover computation. The output data from the Chile-SR model is composed of global horizontal radiation diffuse horizontal radiation and DNI in hourly basis. Figure 6 illustrates the sequence of main steps that Chile-SR takes in order to estimate radiation for January 25, 2001, at 16:40 UTC. It can be seen from the visible channel picture that cloud formations were present in the Pacific Ocean, southern Chile, and also covering a thin strip of territory in northern Chile. Most of the territory was free of cloud covers. This first picture also illustrates an additional difficulty that the research team has faced, in the form of salt lakes and snow covers that in a visible channel picture might appear as cloud covers. The next image, IR channel, complements the visible channel image by giving information about the temperatures of each region. By properly combining the information from both channels, a cloud classification can be made, thus determining if a particular region is clear of clouds (type 1 in the figure), or if it has cloud covers (types 2 to 4 in the figure). The next step is processing the cloud type and comparing the instantaneous information from each image to a monthly-established reference, which

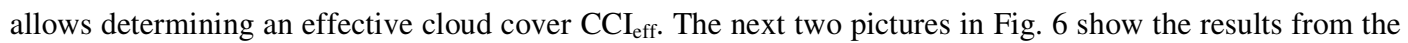
Chile-SR model for both global horizontal irradiance and DNI. It can be observed that northern Chile exhibits a high GHI up to $1200 \mathrm{~W} / \mathrm{m}^{2}$, which steadily decreases with latitude. Cloud covers in northern and southern Chile decrease the GHI down to about $400 \mathrm{~W} / \mathrm{m}^{2}$, the same as along the Andes Mountains. DNI values are also high well in excess of $1000 \mathrm{~W} / \mathrm{m}^{2}$, and are severely diminished by the presence of cloud covers.

From what can be seen in the form of preliminary results, the Chile-SR model is a significant advance in the solar energy resource assessment for Chile, as it is able to capture the different climate conditions present in the country. However, it is necessary to advocate caution as the model is yet to be fully validated; although preliminary comparisons with ground station data are promising, a more detailed analysis is being performed which will reveal both the model potential shortcomings and strengths. In particular, the model aims to capture the cloud-clear conditions present in northern Chile and the cloud variability observed in southern Chile; both conditions have not been fully observed in the time period where both satellite-estimated and ground station data are available, and thus our main hypothesis remain untested. In particular -as a twisted way in which nature enjoys playing with our project schedule- the weather conditions during 2011 have seen many cloudy days and even rain in northern Chile, something that seldom occurs and is considered as one-inhundred-years weather. As mentioned, ground station data is being produced in the UC-FONDEF network since mid-2010, with some stations still to be deployed in their final and definite location. Satellite-estimated data has been produced since May 2011, and thus a first comparison with several months' worth of data is expected to be concluded by the end of September 2011. 


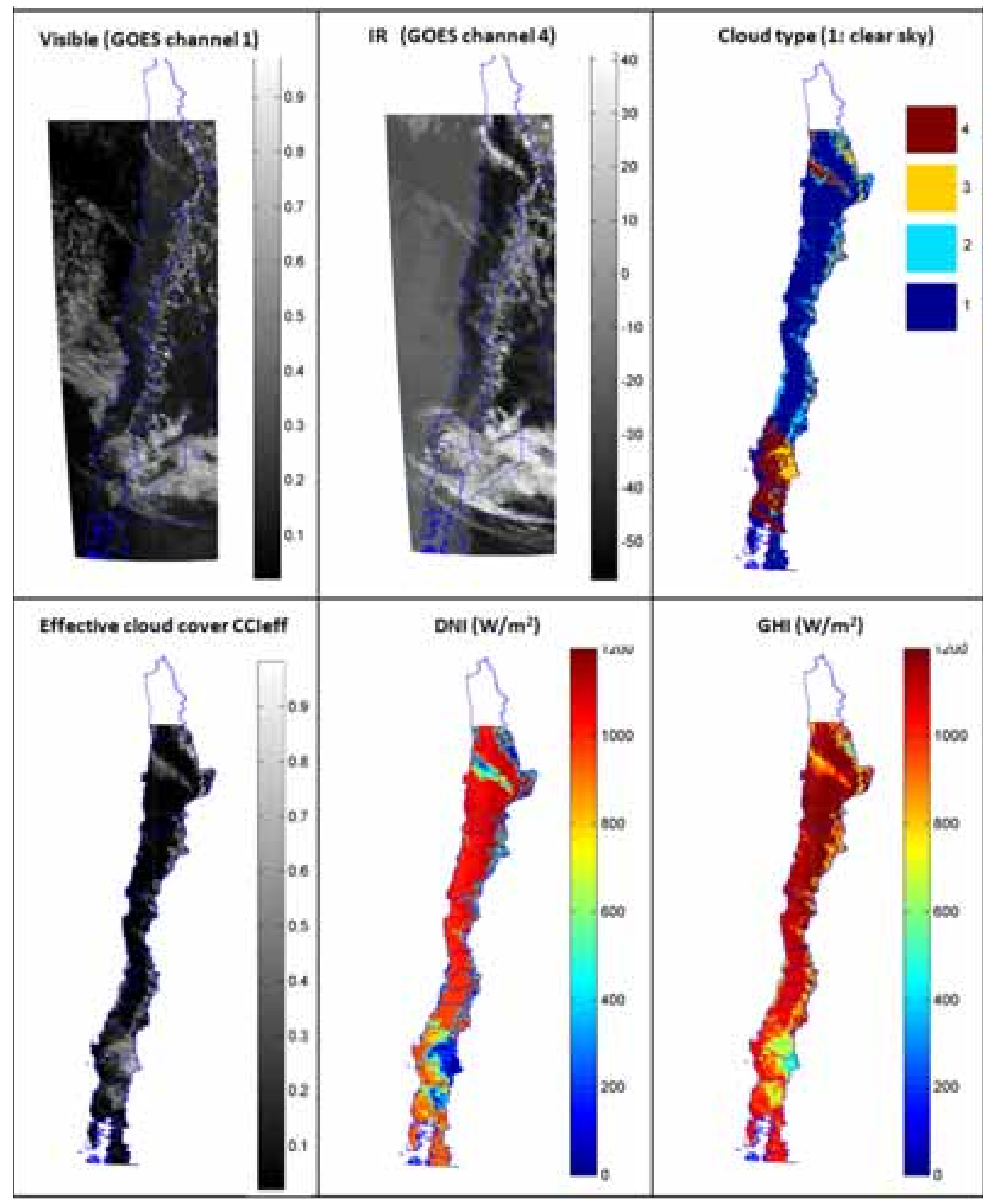

Figure 6: Sequence of steps going from satellite image to radiation estimates with the Chile-SR model, for January 252001 , 16:40 UTC.

\section{Comparing the different sources of data}

The most recent data for Chile, as explained before, is composed of the CNE-GiZ and UC-FONDEF ground stations networks, and the satellite-derived data from Chile-SR. Although it would be highly desirable to perform a direct comparison of all three data sources, the fact that the ground stations from both networks are located in different locations prevents that comparison. However, several comparisons can be attempted in order to assess the weaknesses and strengths of each data source. In what follows, first a comparison of raw and processed data from a CNE-GiZ station is performed. Then, data from relatively closely located stations from the UC-FONDEF and CNE-GiZ networks is compared. Finally, preliminary comparisons of data from 
the Chile-SR model and UC-FONDEF ground station data provide partial validation of the model's performance.

Since a significant part of this report focuses on the CNE-GiZ and UC-FONDEF networks, the latter being deployed and operated by the authors, it is important to establish here that both networks are unrelated. Although a degree of collaboration exists between both operators, their efforts are mostly unrelated and uncoordinated.

\subsection{Comparing raw and processed data from a CNE-GiZ station}

As mentioned in the system description (section 2.3), the CNE-GiZ stations are designed in a non-standard configuration that does not follow international standards or best practices. In addition to the sensor mounting in a one-axis tracker that is used to estimate direct radiation in the tracking plane, a non-standard shadow ring is used, and no correction factor is reported to account for this in the diffuse radiation reading. Additionally, no radiation shields are provided in order to block ground-reflected radiation, and the pyranometers are directly installed on top of a steel plate of high reflectivity that reflect direct radiation to the inner shadow ring and then back to the pyranometers. As stated before, the data is saved in 10-minute intervals, and maintenance is performed on a monthly basis. Thus, the several shortcomings in the design and operation of this station severely restrict the validity of the measurements, to a point in which the data has been sent to ISE Fraunhofer for reprocessing. Unfortunately, the reprocessing methodology has not been made public, although the reprocessed data is available for the period March-September 2010. Figure 7 displays a comparison between raw and processed data for September 21, 2010, where it can be observed the

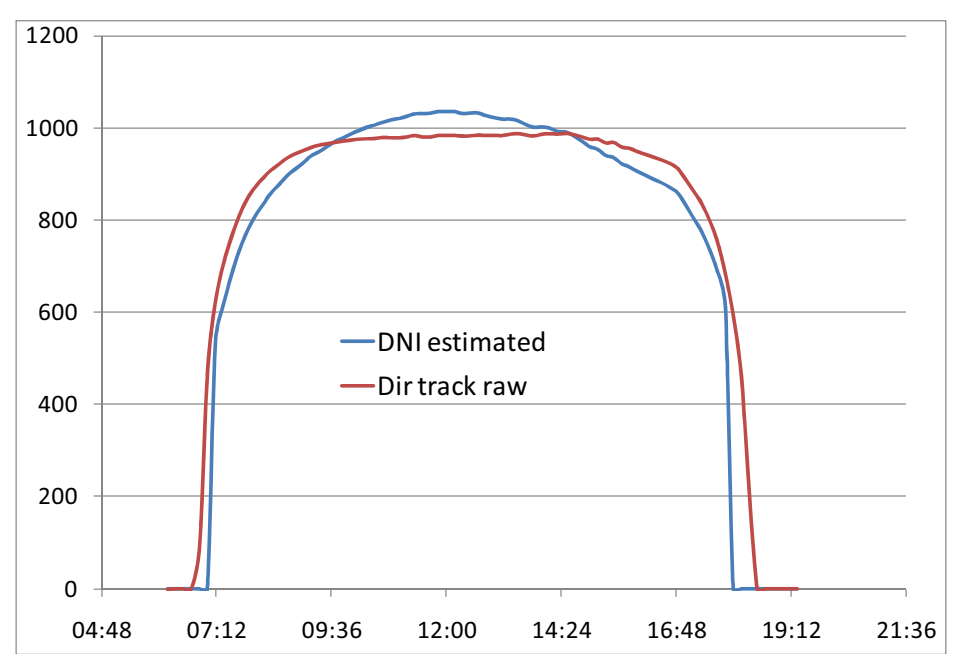
differences that exist in the socalled "Direct irradiation in tracking plane" (DTP) and the estimated DNI (produced from raw data processing by the ISE Fraunhofer)

Figure 7: A comparison of CNE-GiZ data for September 21, 2010.

It can be observed that the DTP displays non-zero values from earlier to later than the estimated DNI. This is due to light being measured before the sun arises

from behind the Andes and after it settles below the horizon. The estimated DNI reaches higher values than the DTP, but an integration of both DNI and DTP over the day yields daily totals within 5\% of each other. Although this could be interpreted as the DTP being an acceptable surrogate for DNI, no formal comparison with pyrheliometer data has been made, and thus it is better to wait for that comparison before reaching a conclusion on the data validity. Moreover, the processed data is only available for 6 months, and thus has not covered a complete year in order to analyze the data behavior for all seasons. While the data processing appears to be an advance that leads to better quality data, it is very difficult to argue in favor or against this procedure without knowing its details; until the procedure for processing the data is made public, one can only guess about its validity and therefore no analysis can be performed other than to say that the data has, in effect, being re-processed.

\subsection{Comparing UC-FONDEF and CNE-GiZ data}

The previous section establishes that since the CNE-GiZ stations are non-standard there are questions about the data quality and validity; this has led the operator to send the data for re-processing in order to establish both the uncertainty of the measurements and to compute an estimation of DNI. Although no comparison 
with pyrheliometer data has been performed for the CNE-GiZ network, one UC-FONDEF station with a pyrheliometer device is located at $8 \mathrm{~km}$ distance from one of them, both in the San Pedro de Atacama town in northern Chile. This distance is thought to be sufficiently close to allow a comparison between data generated by both stations, which could be considered as valid especially in clear days as both stations are located within the same geographical area with one definite weather system. Moreover, they are both located at roughly the same altitude and distance to the Andes Mountains. Figure 8 shows a comparison between GHI produced by the two stations (top), and the DTP compared to DNI. Unfortunately, the pyrheliometer
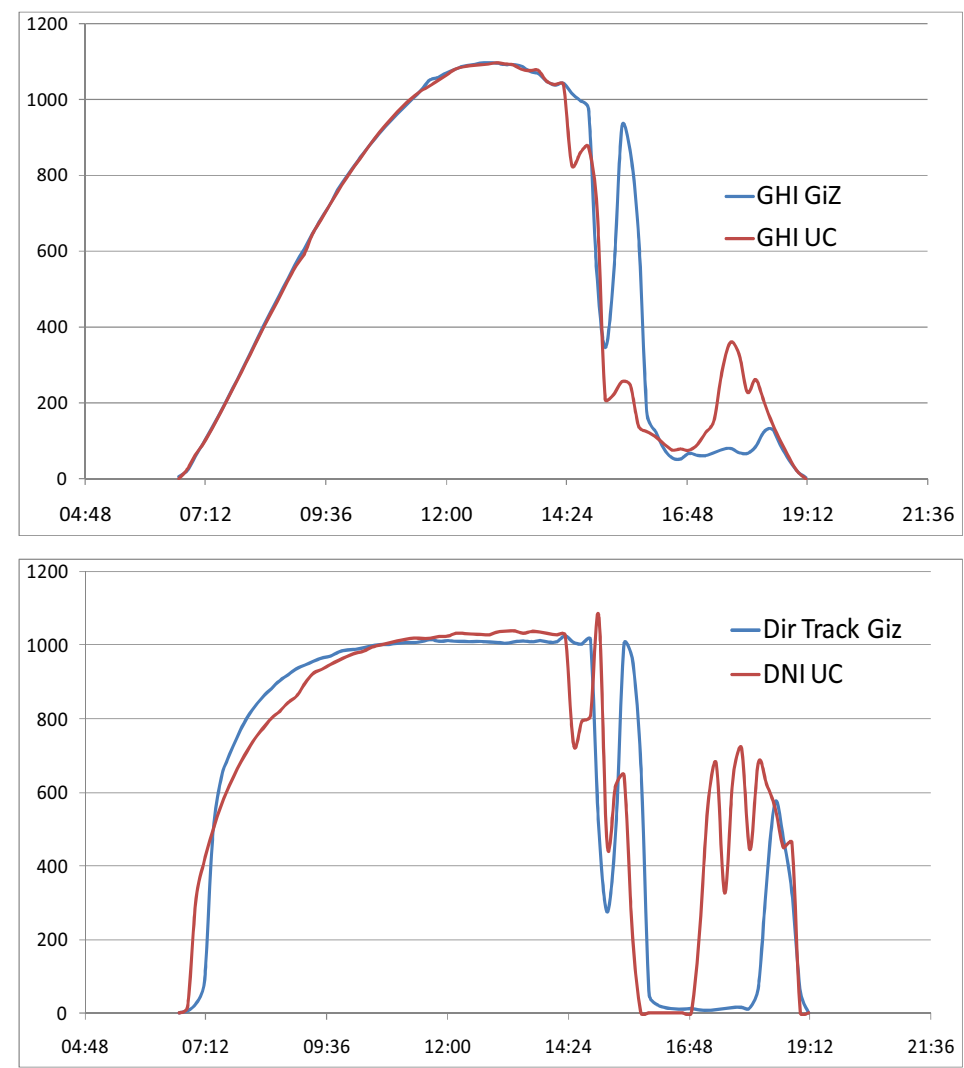

data is only available since December 2010, while the processed data covers marchSeptember 2010 as previously explained. This prevents from performing a complete comparison including DTP, estimated DNI, and measured DNI.

Figure 8: Comparing ground measurements San Pedro de Atacama taken with the UCFONDEF and CNE-GiZ stations for March 1, 2011. Data in $\mathrm{W} / \mathrm{m}^{2}$.Top: Global horizontal irradiance. Bottom: Estimated direct irradiance in tracking mode (CNE-GiZ) and measured DNI (UC-FONDEF)

The day chosen for this comparison is March 1, 2011, a day that started clear and then became partially cloudy. For the GHI, it can be observed that from sunrise until about half an

hour after solar noon, the day remained clear and the data from both stations agrees very well; in fact, the data from each station cannot be distinguished from the other. This is not surprising, since both sensors are the same brand and model and are measuring the same parameter in a geographically similar location. Shortly after solar noon, the data from both stations shows diminishing radiation levels that indicate the presence of scattered clouds. This phenomenon is well known for the area where both stations are located, where these clouds originating in the Andes Mountains travel west, eventually dropping rain in what is known as "Bolivian winter" in reference to the clouds origin over the country of Bolivia, located further east. That the data from both stations from this point on widely differs indicates that the clouds passing over each site are local in nature, and thus the distance between stations (about $8 \mathrm{~km}$ ) results in each being subject to very site-specific local weather conditions. Perhaps the most important conclusion from this comparison is found in the first pat of the day, while both stations are measuring clear-day irradiance. It can be observed that the measured DNI is at first higher than the DTP, then they intersect resulting in the DTP having a higher value, to finally intersect again and the measured DNI being higher again. This behavior is different to the one displayed by the estimated DNI from re-processed data, which only intersects the DTP once. However, regardless of this observed differences, it must be stated that this comparison is far from conclusive, and that therefore it is better to avoid jumping into conclusions other than the three parameters (measured DNI, estimated DNI, and DTP) must be properly compared in order to establish their validity. Such comparison process is being underway in a collaborative effort by GiZ and UC, and the results are 
expected to be available within the next months.

\subsection{Chile-SR satellite-derived data and UC-FONDEF ground stations}

Figure 9 shows the comparison for satellite-derived data and ground measurements including both partiallyclouded and clear days. The satellite-derived data includes both data produced with the original Brasil-SR model and the modified Chile-SR model. Considering the time of the year and the measurement location (Santiago in January, during summertime), it was not possible to obtain data for a fully-clouded day. It can

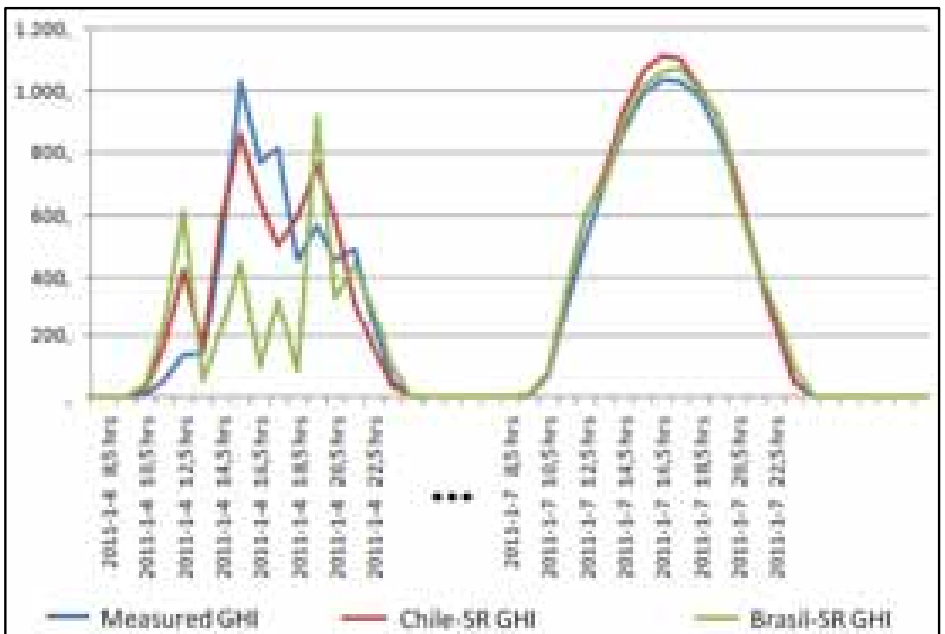
be seen that for a clear day both the Brasil-SR and Chile-SR models slightly overestimate GHI measured data, and that this overestimation is larger with the Chile-SR model.

\section{Figure 9: Comparing Satellite- derived data and ground measurements in Santiago for a cloudy (left) and clear (right) day. GHI data in $\mathrm{W} / \mathrm{m}^{2}$.}

For a cloudy day, however, the situation is very different. Both the Brasil-SR and Chile-SR satellite-based models apparently are not fully capable to estimate the GHI with precision; with the Brasil-SR model severely underestimating the measurements.
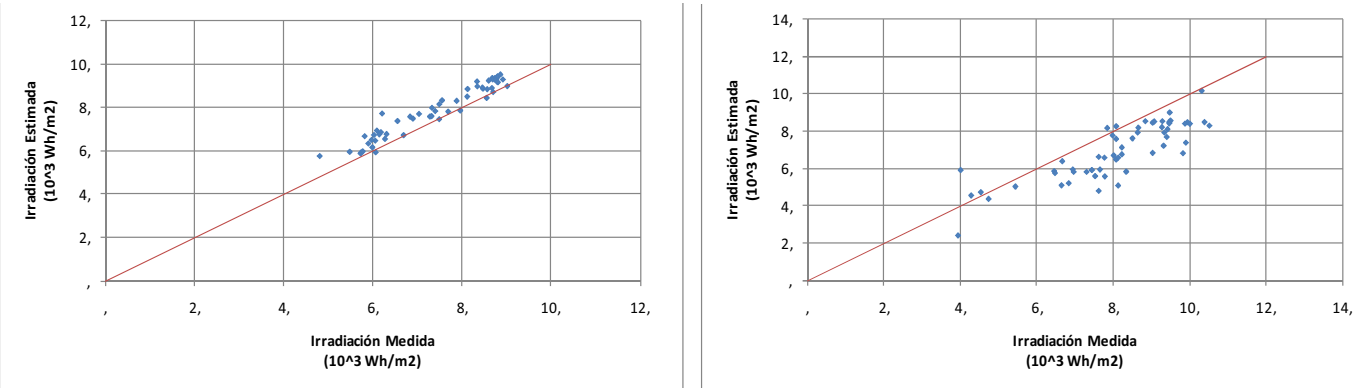

Figure 10: Comparing Satellite-derived data and ground measurements in Santiago for GHI (left) and DNI (right) data in $\mathrm{W} / \mathbf{m}^{2}$. A total of only 57 full days were available for this comparison.

The available number of data samples at time of this reports completion was low (only 57 full days of ground station data), and thus no meaningful comparison can be performed. Moreover, the available measurement data didn't include fully cloudy days, and therefore all comparisons are for days with relatively high radiation. Even then, as a preliminary analysis, it is possible to observe that there are two clear tendencies for the satellite-derived data, as seen in Figure 10, which displays the comparison for Santiago. First, it is possible to observe that the GHI satellite-derived data is higher than measured data, and that the DNI satellite-estimated data is lower than what was measured. Second, the satellite-estimated DNI shows a larger dispersion than the GHI. With such few data points it is very difficult to extract a meaningful conclusion from the comparison, other than say that the Chile-SR model is apparently capturing the radiation behavior with an acceptable error level. However, it is necessary to emphasize that any conclusion is premature, the Chile-SR model is not fully validated, and that the validation process is currently being conducted by UC and INPE researchers. 


\section{Conclusions}

Renewable Energy promotion efforts in Chile aim to achieve a power production quota of $10 \%$ to be met by 2024. This plan has sparked interest in solar energy among other renewable sources, with PV, CSP, and industrial heat supply plants being announced. Previous reports by the authors have identified several databases of solar radiation finding that significant deviation exists between sources, with most data from ground station measurements displaying unknown uncertainty levels. This highlighted the need for a proper, country-wide long-term resource assessment initiative. This report updates the situation in Chile by considering the efforts performed in the last two years by two different resource assessment programs; one by CNE-GiZ and other by UC.

The CNE-GiZ program has expanded its ground station network reaching coverage at seven different locations. The sensor setup in this network has been criticized as being non-standard and therefore the validity of the generated data is not assured; this has led the operator to reprocess the data in order to quantify the uncertainty levels and compute an estimation of DNI thought to be more precise and representative of the actual irradiance conditions than the measured DTP. The UC-FONDEF program is deploying a network of five BSRN-designed and seven RSBR devices, which are producing data according to international standard and good practices. Both networks are relatively new, and the oldest station has been producing data for less than three years. A satellite estimation model has been developed to account for the particular conditions found in Chile, which is already producing data and it is currently being validated. Preliminary data from the Chile-SR model indicates good agreement with ground measurements, although this preliminary validation is by no means exhaustive and requires further work.

As seen, Chile still has no database of solar radiation data that could be considered as valuable for project design and financing activities. However, the current efforts are starting to yield interesting results by producing high quality data that is expected to be released to the public shortly. It is then expected by the authors that the lack of solid data for Chile is about to be solved.

\section{Acknowledgements}

The authors would like to gratefully acknowledge financial support from FONDEF through project grant D08i1097. Prof. Andrés Olivares from UTFSM supplied the NSA data. Karin Franzen and Trudy Konnemund of GiZ supplied data from their stations and were open for discussions. Ed Kern of Irradiance Inc. kindly helped us master the subtleties of the RSBR units.

\section{References}

Pereira, E.; Martins, F.; de Abreu, S.; Ruther, R. Atlas Brasilero de Energía Solar. INPE, 2006.

Perez, R.; Seals, R.; Zelenka, A. Comparing Satellite Remote Sensing and Ground Network Measurements for the Production of Site/Time Specific Irradiance Data. Solar Energy 1997; 60 (2): 89-96.

Zelenka, Pérez, Seals and Renne, Effective accuracy of satellite-derived hourly irradiance. Theoretical and Applied Climatology 62: 199-207, 1999.

R. Pitz-Paal, Norbert Geuder, Carsten Hoyer-Klick, Christoph Schillings. How to get bankable meteo data? DLR solar resource assessment. NREL 2007 Parabolic Trough Technology Workshop, March 8-9, 2007, Golden, Colorado. Last accessed at http://www.nrel.gov/csp/troughnet/wkshp_2007.html on Dec. 29, 2009.

Pacific Northwest Solar Radiation Data book. University of Oregon Solar Monitoring Laboratory, 1999. Last accessed at http://solardat.uoregon.edu on on Dec. 29, 2009.

Cáceres, R. Modelo Estadístico de la Radiación total en plano horizontal para diversas estaciones del país. Memoria de Título; Facultad de Ingeniería, Departamento de Mecánica, Universidad Técnica Federico Santa María, Chile 1984.

Ortega, A., Escobar, R., Colle, S., Abreu, S. (2010). The State of Solar Energy Resource Assessment in Chile. Renewable Energy, 35, 11, 2514-2524. 